http://dx.doi.org/10.35381/s.v.v5i1.1597

\title{
Risoterapia dentro de los cuidados de enfermería en pacientes pediátricos
}

Laughter therapy as part of nursing care for pediatric patients

\author{
Adisnay Rodríguez-Plasencia \\ ua.adisnayrodriguez@uniandes.edu.ec \\ Universidad Regional Autónoma de los Andes, Ambato \\ Ecuador \\ https://orcid.org/0000-0002-5293-2817 \\ Ingrid Valeria Flores-Zambrano \\ ea.ingridvfz30@uniandes.edu.ec \\ Universidad Regional Autónoma de los Andes, Ambato \\ Ecuador \\ https://orcid.org/0000-0002-7079-5625 \\ Crislyn Vanessa Remache-Gamarra \\ ea.crislynvrg53@uniandes.edu.ec \\ Universidad Regional Autónoma de los Andes, Ambato \\ Ecuador \\ https://orcid.org/0000-0002-7079-5625
}

Recepción: 10 de agosto 2021

Revisado: 15 de septiembre 2021

Aprobación: 15 de noviembre 2021

Publicación: 01 de diciembre 2021 


\section{Sra. Editora:}

Como futuros profesionales de Enfermería sabemos que la hospitalización de un paciente pediátrico implica someterse a un mundo desconocido, el mismo hace que los niños se sientan física y emocionalmente mal al enfrentarse a procedimientos invasivos dolorosos. Por lo tanto, es importante es enfatizar en la importancia de la risoterapia (1) (2) (3), por cuanto; es una de las múltiples terapias complementarias que el personal de enfermería usa para la recuperación del paciente pediátrico de manera integral tras someterse al estrés de una hospitalización.

Esta terapia posee múltiples beneficios, descubrieron que reír de forma hilarante reducía los niveles de estrés y que la risa tiene un efecto sobre el sistema inmunitario, la Risoterapia, como complemento al tratamiento convencional, es una técnica que podría mejorar el estado de salud de un paciente (4) (5) (6). Por consiguiente, el personal de enfermería debe considerar en los cuidados que proporciona del dolor mediante escalas y así aplicar la risoterapia, ya que esta terapia costo-efectiva, muy fácil de aplicarla y mejorar la calidad de vida tanto del paciente como de su entorno y estudios existentes muestran múltiples beneficios de calidad.

En consecuencia, la risoterapia dentro del contexto de la salud es un instrumento poderoso de curación que fortalece el sistema inmunológico del cuerpo, además es considerada como un procedimiento alternativo, complementario y preventivo, que proporciona al organismo beneficios a nivel fisiológico, psicológico y espiritual que se puede realizar con todo tipo de pacientes con patologías agudas o crónica. Siendo necesario efectuar investigaciones con una estructura metodológica más sólida que permita sustentar su aplicabilidad de esta terapia y por lo expuesto anteriormente, sin embargo, por los datos apriorísticos existentes hasta ahora, se sugiere que la risoterapia debe ser incorporada en el ámbito de la salud como un medio útil y saludable en el tratamiento de enfermedades. 
Revista Arbitrada Interdisciplinaria de Ciencias de la Salud. SALUD Y VIDA

Volumen 5. Número 1. Año 5. Especial. 2021

Hecho el depósito de Ley: FA2016000010

ISSN: $2610-8038$

FUNDACIÓN KOINONIA (F.K)

Santa Ana de Coro, Venezuela.

Adisnay Rodríguez-Plasencia; Ingrid Valeria Flores-Zambrano; Crislyn Vanessa Remache-Gamarra

\section{CONFLICTO DE INTERÉS}

Los autores declaran que no tienen conflicto de interés en la publicación del artículo.

\section{FINANCIAMIENTO}

No monetario.

\section{AGRADECIMIENTO}

A la Universidad Regional Autónoma de los Andes, por apoyar el desarrollo de la investigación.

\section{REFERENCIAS}

1. Sridharan K, Sivaramakrishnan G. Therapeutic clowns in pediatrics: a systematic review and meta-analysis of randomized controlled trials [published correction appears in Eur J Pediatr. 2017;176(5):681-682]. Eur J Pediatr. 2016;175(10):13531360. doi:10.1007/s00431-016-2764-0

2. Devictor D. Les clowns en pédiatrie [Clowns in pediatrics]. Arch Pediatr. 2015;22(1):1-3. doi:10.1016/j.arcped.2014.09.017

3. Dionigi A. Clowning as a Complementary Approach for Reducing latrogenic Effects in Pediatrics. AMA J Ethics. 2017;19(8):775-782. doi:10.1001/journalofethics.2017.19.8.stas1-1708

4. Kuru N, Kublay G. The effect of laughter therapy on the quality of life of nursing home residents. J Clin Nurs. 2017;26(21-22):3354-3362. doi:10.1111/jocn.13687

5. Effects of Laughter Therapy on Life Satisfaction and Loneliness in Older Adults Living in Nursing Homes in Turkey: A Parallel Group Randomized Controlled Trial. Rehabil Nurs. 2021;46(2):E10-E11. doi:10.1097/RNJ.0000000000000321

6. Lopes-Júnior LC, Bomfim E, Olson K, et al. Effectiveness of hospital clowns for symptom management in paediatrics: systematic review of randomised and nonrandomised controlled trials. BMJ. 2020;371:m4290. doi:10.1136/bmj.m4290 
Revista Arbitrada Interdisciplinaria de Ciencias de la Salud. SALUD Y VIDA

Volumen 5. Número 1. Año 5. Especial. 2021

Hecho el depósito de Ley: FA2016000010

ISSN: $2610-8038$

FUNDACIÓN KOINONIA (F.K)

Santa Ana de Coro, Venezuela.

Adisnay Rodríguez-Plasencia; Ingrid Valeria Flores-Zambrano; Crislyn Vanessa Remache-Gamarra

2021 por los autores. Este artículo es de acceso abierto y distribuido según los términos y condiciones de la licencia Creative Commons Atribución-NoComercial-Compartirlgual 4.0 Internacional (CC BY-NC-SA 4.0) (https://creativecommons.org/licenses/by-nc-sa/4.0/). 